\title{
Role of the hypothalamic pituitary adrenal axis in the control of the response to stress and infection
}

\section{S.M. McCann ${ }^{1}$, \\ J. Antunes-Rodrigues², \\ C.R. Franci ${ }^{2}$, \\ J.A. Anselmo-Franci ${ }^{3}$, \\ S. Karanth ${ }^{1}$ and \\ V. Rettori ${ }^{4}$}

\author{
1Pennington Biomedical Research Center (LSU), Baton Rouge, LA, USA \\ Departamentos de Fisiologia da ${ }^{2}$ Faculdade de Medicina e da \\ ${ }^{3}$ Faculdade de O dontologia de Ribeirão Preto, Universidade de São Paulo, \\ Ribeirão Preto, SP, Brasil \\ ${ }^{4}$ Centro de Estudios Farmacologicos y Botanicos, Consejo Nacional de Investigaciones \\ Cientificas y Tecnicas (CEFYBO-CONICET), Buenos Aires, Argentina
}

\section{Correspondence \\ S.M. McCann \\ Pennington Biomedical Research \\ Center (LSU) \\ Baton Rouge, LA 70808-4124 \\ USA \\ Fax: + 1-225-763-3030 \\ E-mail: mccannsm@ mhs.pbrc.edu \\ Presented at the First \\ International Meeting on Adrenal \\ Disease: Basic and Clinical \\ Aspects, Ribeirão Preto, SP, Brazil, \\ August 31-September 2, 1999.}

Research supported by the National Institutes of Health (N OS. DK43900 and MH51853) to S.M. McCann. Publication supported by FAPESP.

Received December 20, 1999 Accepted March 10, 2000

\section{Abstract}

The release of adrenocorticotropin (ACTH) from the corticotrophs is controlled principally by vasopressin and corticotropin-releasing hormone (CRH). Oxytocin may augment the release of ACTH under certain conditions, whereas atrial natriuretic peptide acts as a corticotropin release-inhibiting factor to inhibit ACTH release by direct action on the pituitary. Glucocorticoids act on their receptors within the hypothalamus and anterior pituitary gland to suppress the release of vasopressin and CRH and the release of ACTH in response to these neuropeptides. CRH neurons in the paraventricular nucleus also project to the cerebral cortex and subcortical regions and to the locus ceruleus (LC) in the brain stem. Cortical influences via the limbic system and possibly the LC augment CRH release during emotional stress, whereas peripheral input by pain and other sensory impulses to the LC causes stimulation of the noradrenergic neurons located there that project their axons to the $\mathrm{CRH}$ neurons stimulating them by $\alpha$-adrenergic receptors. A muscarinic cholinergic receptor is interposed between the $\alpha$-receptors and nitric oxidergic interneurons which release nitric oxide that activates $\mathrm{CRH}$ release by activation of cyclic guanosine monophosphate, cyclooxygenase, lipoxygenase and epoxygenase. Vasopressin release during stress may be similarly mediated. Vasopressin augments the release of CRH from the hypothalamus and also augments the action of $\mathrm{CRH}$ on the pituitary. CRH exerts a positive ultrashort loop feedback to stimulate its own release during stress, possibly by stimulating the LC noradrenergic neurons whose axons project to the paraventricular nucleus to augment the release of $\mathrm{CRH}$.

\section{Introduction}

Dramatic progress has been made in peptide research over the last 45 years, and we now know the specific hypothalamic peptides that control the release of various pitu-

\section{Key words}

- Corticotropin-releasing

hormone (CRH)

- Vasopressin

- Oxytocin

- Atrial natriuretic peptide (ANP)

- ACTH

- Cortisol

- Norepinephrine

- Acetyl choline

- Nitric oxide itary hormones. In the mid-1940's it was recognized that neural stimuli could evoke release of hormones from the anterior pituitary. Examples included coitus-induced ovulation in birds, ferrets, cats, and other mammals, suckling-induced prolactin (PRL) re- 
lease, and stress-induced release of adrenal corticotropic hormone (ACTH). One of the most important discoveries in the field was that of the hypophyseal portal system of veins by Popa and Fielding in 1933. These originate from capillaries in the median eminence (ME), drain blood down the hypophyseal stalk, and supply the sinusoids of the anterior lobe of the pituitary gland. Although blood flow was initially thought to be upward in these vessels, Houssay and his colleagues observed downward flow in the living toad in 1935. This observation was confirmed in the living rat by Green and Harris in 1947. It is apparent then that the primary flow is downward from the ME to the pituitary; however, there is evidence that upward flow may indeed occur under certain conditions.

In the meantime, it became apparent that there was little if any functional innervation to the anterior lobe, prompting a number of workers to suggest that hypothalamic control over the pituitary might be mediated via neurohumoral agents released into the capillaries of the hypophyseal portal system that would pass down the pituitary stalk and stimulate the release of particular pituitary hormones from the cells in the gland specialized to produce each hormone. Indeed, deficits in pituitary hormone secretion followed lesions placed in various hypothalamic loci, and conversely, electrical stimulation of these same regions was shown to evoke release of gonadotropins and ACTH. That the portal system was a crucial link between brain and pituitary was suggested by the experiments of Harris and his collaborators who showed that following stalk section the portal vessels frequently regenerated, and a return of normal pituitary function accompanied this regeneration. On the other hand, a plate placed between the cut ends of the stalk to block such regeneration caused a permanent impairment in pituitary function. In other experiments it was shown that if the pituitary was removed from its capsule and then im- mediately transplanted under the ME, so that it was vascularized by the portal vessels, pituitary function returned to normal. A corollary of this experiment was carried out by Nikovitch-Winer and Everett who showed that pituitary grafts under the kidney capsule did not sustain normal pituitary function but later regrafting under the ME returned function to normal (for reviews, see Refs. 1 and 2).

\section{Corticotropin-releasing factors}

The next logical step was to make extracts of the ME and to evaluate their effects on pituitary hormone secretion. Because of the early development of sensitive assays for determining ACTH release, attention first focused on the ability of such extracts to evoke ACTH release. Because of the ready availability of partially purified extracts of the posterior pituitary, which were used in the preparation of commercial vasopressin (Pitressin) and oxytocin (Pitocin), these extracts were tested in early experiments. To make extracts of the stalk ME required laborious collection of the tissue at the slaughterhouse. The initial choice of posterior pituitary extracts for testing was unfortunate but led to the discovery of the ACTH-releasing activity of vasopressin (3). Just after this, vasopressin was synthesized by Du Vigneaud and colleagues (see Ref. 4), and the synthetic material was shown to be active in vivo to release ACTH in animals with ME lesions that blocked the ubiquitous release of ACTH from stress. It was reported that a substance different from vasopressin could be isolated from posterior pituitary extracts that also released ACTH; this substance was named corticotropin-releasing factor (CRF) $(5,6)$. Early claims for a CRF other than vasopressin in posterior pituitary extracts have not been confirmed $(7,8)$. Guillemin and Schally even claimed to have partial structures of $\beta C R F$ and $\alpha$ I and II CRF. Again, these claims have not been confirmed (8). At 
this point in time, it is clear that there is such a factor in posterior lobe extracts; however, it is a minor contaminant and the major ACTH-releasing activity of such extracts is accounted for by vasopressin $(8,9)$.

It was only when attention was turned to extracts of the stalk ME that it became clear that indeed there was a separate corticotropin-releasing hormone (CRH) different from vasopressin (9). This material was purified and separated from vasopressin (10), but its isolation and determination of structure proved difficult. In fact, although CRH was the first releasing factor to be discovered, it was one of the last whose structure was elucidated, probably because it was a much larger polypeptide. This feat was finally accomplished by Vale and Rivier and their colleagues in 1981 (11). CRH turned out to be a 41 -amino acid peptide, completely distinct from the octapeptide vasopressin, and with no disulfide group in the molecule.

It is now clear that vasopressin and $\mathrm{CRH}$ share physiological roles as CRFs. The vasopressin neuronal system has cell bodies primarily in the supraoptic nucleus (SON) but also in the paraventricular nucleus (PVN) and axons that project primarily to the neural lobe; however, some of these axons, particularly those from the PVN, terminate in the external layer of the ME in juxtaposition to portal vessels. High concentrations of vasopressin and CRH are found in the portal vessels, and both peptides release ACTH in concentrations similar to those found in portal blood (12). There is a defect, albeit small, in ACTH secretion in animals with hereditary diabetes insipidus that lack vasopressin secretion (13). Vasopressin can potentiate the response to $\mathrm{CRH}$ at the pituitary level as revealed by both in vivo (14) and in vitro experiments (15). The peptide is also active in the hypothalamus to potentiate the release of CRH (16). Antisera directed against vasopressin injected into the third ventricle produce a partial block in ACTH secretion, and a partial block has also been achieved with vasopressin antagonists (17). There are vasopressin type II receptors similar to those in the vascular system on anterior pituitary corticotrophs that act to increase intracellular calcium in these cells (12), inducing a release of ACTH. On the other hand, $\mathrm{CRH}$ acts on CRH type I receptors on the corticotroph to activate adenylyl cyclase, leading to an increase in cyclic adenosine monophosphate (cAMP) that induces ACTH release (12).

An early candidate for CRF was epinephrine; however, its direct CRF activity appeared to be ruled out by the ability of ME lesions to block the response to epinephrine. This early concept is now reemerging in view of the ability of epinephrine to evoke ACTH release both in vivo and in vitro by an action on B-receptors which have been discovered in the gland. Epinephrine is released during stress and could activate ACTH secretion either by the increased concentrations in the circulation or by its release into portal blood, which would provide much higher concentrations of the catecholamine. In fact, high concentrations of epinephrine have recently been reported in portal blood. From all of this, it is apparent that there is no single CRF. Instead there is a constellation of agents that can release ACTH and may play physiological roles in evoking the stressinduced release of the peptide (12).

The perikarya of CRH and vasopressin neurons are located predominately in parvocellular neurons of the SON and PVN. The preponderance of vasopressin neurons is in the SON and of CRH neurons in the PVN. The axons project to the ME where the preponderance of vasopressin axons continues into the neural lobe to release the peptide there. The preponderance of CRF neurons terminates in the external layer of the ME and only a few project to the neural lobe. Early studies indicated that lesions in the ME that induce severe diabetes insipidus block ACTH secretion, presumably because they block these axons of vasopressin and 
CRH neurons reaching the ME from both the PVN and SON (3). Lesions of the PVN did not block the stress response when studied 2 to 3 weeks later, but were associated with adrenal atrophy (3); however, later studies showed that lesions of the PVN were effective to block ACTH secretion when tested only 5 days after lesions (18). These experiments support the concept that both PVN and $\mathrm{SON}$ are involved in $\mathrm{ACTH}$ secretion by release of $\mathrm{CRH}$ and vasopressin, the former into the hypophyseal portal vessels in the $\mathrm{ME}$ and the latter also into the short portal vessels in the neural lobe. These supply $1 / 3$ of the blood to the anterior lobe and probably play an important role in control of anterior lobe function through release of hypothalamic factors into these vessels, in particular, vasopressin in the case of ACTH secretion, a fact that has been largely ignored.

Some of the CRH neurons in the PVN projecting to the ME also contain vasopressin, and the relative abundance of vasopressin is increased in the adrenalectomized animal. Therefore, vasopressin appears particularly to be important in the increase in $\mathrm{ACTH}$ secretion that occurs following adrenalectomy. Indeed, vasopressin appears to be more important than $\mathrm{CRH}$ in control of ACTH secretion in sheep. The relative importance of vasopressin and CRH appears to vary depending on the stress imposed and the details of this relationship have yet to be fully determined. As pointed out above, the two synergize to stimulate ACTH release (see Ref. 17 for a review).

Oxytocin, another peptide released from the magnocellular neurons in the PVN and $\mathrm{SON}$, is also released from axonal terminals in juxtaposition to the portal vessels. It is present in high concentrations in portal blood and also has the capacity to potentiate the response to CRH at the pituitary level. Since oxytocin is released in stress as well as vasopressin and CRF, it may play a small role in the stress-induced release of ACTH (19).
Similarly, angiotensin II neurons within the hypothalamus that project to the ME may play a small augmenting role since the peptide is present in portal blood and has an intrinsic capacity to stimulate the corticotrophs (20). There are also receptors for this peptide on pituitary cells. Angiotensin II is an important hormone that is released in states of reduction of the circulating blood volume and that is associated with $\mathrm{ACTH}$ release (20). Atrial natriuretic peptide (ANP) has opposite effects; namely it is released in states of volume expansion and causes diuresis and natriuresis $(20,21)$. ANP also acts centrally to inhibit vasopressin secretion and has now been clearly shown to have an inhibitory action to suppress CRH release intrahypothalamically and inhibit the pituitary's response to CRH, leading some to call it a corticotropin release-inhibiting factor $(20,21)$.

Many of these actions of angiotensin II and ANP are physiologically significant as revealed by injection of antisera against these peptides into the third cerebral ventricle $(19,22)$. ANP is also synthesized in the anterior pituitary gland and there are receptors in the gland to mediate its action (22).

The principal ANP receptor is guanylyl cyclase. It causes conversion of guanosine triphosphate into cyclic guanosine monophosphate (cGMP) that induces a decrease in intracellular free calcium $\left(\mathrm{Ca}^{2+}\right)$ in the corticotroph, thereby inhibiting ACTH secretion (Antunes-Rodrigues J, Yu WH and McCann SM, unpublished results). Interestingly, neural nitric oxide (NO) synthase (nNOS) is also localized in pituitary cells. When activated by transmitters it produces an increase in intracellular free $\mathrm{Ca}^{2+}$ in the pituitary cells containing the enzyme that generates NO by conversion of arginine in the presence of oxygen and various cofactors. This NO diffuses to the corticotroph and activates guanylyl cyclase, leading to increased cGMP formation that decreases intracellular free $\mathrm{Ca}^{2+}$, thereby inhibiting 
ACTH secretion. Thus, agents that generate $\mathrm{NO}$ in the pituitary, such as cytokines would be expected to suppress rather than stimulate ACTH secretion. Indeed, experiments have indicated that NO will suppress ACTH secretion by direct action on the gland and it has been shown that increased NO will inhibit the stimulatory effect of vasopressin on ACTH secretion (23).

\section{Control of vasopressin CRH and ACTH secretion by negative feedback of cortical steroids}

It was shown by Ingle in 1936 that there was a negative feedback action of adrenal cortical steroids to suppress adrenal cortical hypertrophy following unilateral adrenalectomy. This action is mediated by a direct suppression of the response of the corticotroph to vasopressin (24) and CRH (25). The mechanism of this has yet to be fully elucidated. Furthermore, there is also an action of glucocorticoids on the hypothalamus to suppress the release not only of vasopressin but also of CRH. Following adrenalectomy the release of vasopressin and $\mathrm{CRH}$ is increased and vasopressin appears to be colocalized with $\mathrm{CRH}$ in more neurons than in the intact animal. The actions at both the pituitary and hypothalamic levels are mediated by glucocorticoid receptors which have been localized in the pituitary gland and also in the region of the $\mathrm{CRH}$ neurons in the PVN (26).

\section{Distribution and control of CRH neurons by intra- and extra- hypothalamic neuronal systems}

Not only do vasopressinergic and CRHergic neurons project to the ME and neural lobe of the pituitary gland, but also they project to cortical and subcortical structures and caudally to the brain stem (26). Therefore, it is not surprising that input from the cerebral cortex via limbic system areas, as occurs for example in emotional arousal, can activate $\mathrm{CRH}$ release followed by ACTH and cortical hormone secretion $(27,28)$. The mechanism of this pathway is not known; however, this may be by activation of locus ceruleus (LC) neurons and other brain stem noradrenergic (NAergic) neurons that are known to project NAergic axons to the PVN. Indeed, lesions of the LC will temporarily completely block the release of ACTH induced by ether anesthesia in the rat (29).

Indeed, the LC might be characterized as a head ganglion of the sympathoadrenal system, so that it is activated by stressful stimuli in the periphery and by afferent input by pain and other sensory modalities (29). It also can be stimulated by activation from the cerebral cortex via the limbic system (30). The result is not only activation of $\mathrm{CRH}$ release by projections to the $\mathrm{CRH}$ neurons in the PVN, but also activation of the peripheral sympathoadrenomedullary system by producing a massive discharge of sympathetic terminals into the vascular blood system and also on the adrenal medulla producing what Cannon called the flight or fight response. With the realization that the hypothalamic pituitary adrenal (HPA) axis also participated in this response, interest in the activation of the peripheral sympathoadrenomedullary system has waned; however, evidence that has accrued in recent years indicates that there is a direct sympathetic innervation of the adrenal cortex and that adrenomedullary rests are present in the adrenal cortex. Furthermore, catecholamines can stimulate release of adrenocortical steroids from the ACTH-primed adrenal cortex. Indeed, in chronic stress the continued activation of the sympathoadrenomedullary system sensitizes the cortex to the action of CRH and can even activate the cortex directly. Therefore, continued activation of the sympathoadrenomedullary system can contribute to the increased adrenal cortical steroid production in chronic stress, even though the elevated plasma cortical hormone levels will inhibit 
the response of the pituitary to $\mathrm{CRH}$ and also diminish the release of CRH into the hypophyseal portal vessels (31).

\section{Ultrashort loop positive feedback of CRH to stimulate its own secretion}

Microinjection of tiny amounts of CRH into the third ventricle that had no effect on plasma ACTH resulted in a rapid augmentation of the ACTH release in response to ether stress (32). Therefore, it appears that an ultrashort loop positive feedback of $\mathrm{CRH}$ exists to augment its own secretion that operates only during stress. The site of action of CRH to augment its own release has not been determined. However, this may be mediated by the stimulatory effect of the LC on $\mathrm{CRH}$ release since the intraventricularly injected CRH would reach the LC and might stimulate it to send NAergic input into the CRH neurons, thereby stimulating additional $\mathrm{CRH}$ release. Further experiments need to be performed to determine if this is the mechanism by which CRH augments its own release. The positive feedback of CRH to augment its own release appears to be physiologically significant since antiserum against $\mathrm{CRH}$ injected into the third ventricle augmented ACTH release during stress (33).

\section{Intrahypothalamic action of CRH to inhibit growth hormone (GH) and luteinizing hormone (LH) release}

Not only does $\mathrm{CRH}$ augment its own release by an ultrashort loop positive feedback, but it also can inhibit $\mathrm{GH}$ and $\mathrm{LH}$ release following its intraventricular injection (33-36). It is known that in the rat stress inhibits $\mathrm{GH}$ and $\mathrm{LH}$ release $(33,37)$ and this intrahypothalamic action of CRH may play an important physiological role in inhibiting the release of these hormones, thereby inducing the profile of release of these hormones which occurs in stress. In essence in the rat all stresses, if severe enough, will inhibit $\mathrm{GH}$ and $\mathrm{LH}$ release and this probably has a protective value by turning off reproductive function and growth to mobilize all of the resources of the body to combat the imposed stress.

\section{Role of the hypothalamic pituitary adrenal system in stress}

Stress may be defined as any stimulus that distorts homeostasis in the organism. Therefore, stress can be physical as in the case of excessive heat or cold, trauma, surgical operations or infection or it can be emotional and psychological. Selye, who coined the term, even talked about the stress of life itself. It is the stresses of life that contribute to degenerative disease and old age (38).

Stress can affect every organ system in the body, but the principal mediators of the response to stress are the sympathetic nervous system and the HPA axis. Various hypothalamic releasing and inhibiting hormones are released from their axon terminals in the ME into the capillaries of the hypophyseal portal veins that transport them to the anterior lobe of the pituitary gland. There they stimulate or inhibit the release of the various pituitary hormones that act either directly on body tissues or on their target glands, which in turn release hormones that act on various tissues of the body. Examples of the latter are ACTH, follicle-stimulating hormone (FSH), LH and thyroid-stimulating hormone (TSH), and of the former, are PRL and GH.

Physiological stress is presumably mediated by anxiety recognized in the cerebral cortex that stimulates limbic system structures and probably the $\mathrm{LC}$ and other brain stem NAergic neurons that activate $\mathrm{CRH}$ release by NAergic axons which terminate in the vicinity of CRH neurons in the PVN. Vasopressin release is also stimulated; however, this pathway has not been elucidated. In the case of physical stress, such as pain, cold, heat or trauma, there is afferent input by sensory neurons to the brain stem again 
activating the $\mathrm{LC}$ that then triggers $\mathrm{CRH}$ and probably vasopressin secretion.

\section{The role of NO in the stress response}

In the dozen years since the discovery of $\mathrm{NO}$ in the body and the enzymes forming it, it has become evident that NO plays a pivotal role in the function of every organ system of the body. In this brief review, we will first examine the role of NO under physiological conditions and then indicate how this role is affected by stress. In this connection, most attention has focused on the role of NO in the induction of the responses to infection. Little attention has been given so far to its role in psychological and other types of stress.

Nearly all types of stress elicit a stereotyped pattern of pituitary hormone secretion in humans which consists of a rapid increase in ACTH, PRL and GH secretion (in the rat $\mathrm{GH}$ secretion is inhibited) and inhibition of the secretion of LH, TSH and to a lesser extent FSH. The pattern is caused primarily by alterations in the secretion of hypothalamic releasing and inhibiting hormones.

\section{The role of the hypothalamic pituitary adrenal system in infection}

The hypothalamic-pituitary response to infection can be mimicked by the injection of bacterial lipopolysaccharide (LPS) $i v$ or $i p$. This induces an identical pattern of pituitary hormone secretion as that seen in infection. There is a very rapid increase in plasma ACTH and PRL within a few minutes following $i v$ injection of LPS. The response is dose-related and is accompanied by a rapid inhibition of LH and TSH but not FSH secretion. GH secretion is stimulated in humans but suppressed in the rat (37).

Recent work indicates that central nervous system infection is a powerful inducer of cytokine production in glia and neurons of the brain, which causes induction of inducible NOS (iNOS) and production of poten- tially toxic quantities of NO. Following $i v$ injection of an intermediate dose of LPS, there was an induction of IL (interleukin)- $1 \alpha$ immunoreactive neurons in the preoptic-hypothalamic region (37). These cells were shown to be neurons by the fact that double staining revealed the presence of neuron specific enolase. The neurons were found in saline-injected control animals suggesting that they are normally present, but they increased in number by a factor of 2 within $2 \mathrm{~h}$ following injection of LPS. They are located in a region which also contains the thermosensitive neurons. They may be the neurons that are stimulated to induce fever following injection of LPS. They have short axons that did not clearly project to the areas containing the various hypothalamic releasing and inhibiting hormones, but they could also be involved in the stimulation or inhibition of their release, which occurs following infection.

This study led to further research which demonstrated that ip injection of a moderate dose of LPS induced IL-1ß and iNOS mRNA in the brain, anterior pituitary and pineal glands. The results were very exciting since an induction of IL- $1 B$ and iNOS mRNA occurred with the same time-course as found in the periphery following injection of LPS, namely, clear induction of IL-1ß followed by iNOS mRNA within $2 \mathrm{~h}$ reaching a peak in 4-6 $\mathrm{h}$, followed by a decline to near basal levels at the next measurement by $24 \mathrm{~h}$ after the injection. The induction of both mRNAs occurred in the meninges, the choroid plexus, the circumventricular organs, such as the subfornical organ and ME, in the ependymal cells lining the ventricular system, and very suprisingly in parvocellular neurons of the PVN and arcuate nucleus (AN), areas of particular interest since they contain the hypothalamic releasing and inhibiting hormone producing neurons and also other neurotransmitters controlled by NO (39).

The greatest induction occurred in the anterior lobe of the pituitary, where the iNOS 
mRNA was increased at $2 \mathrm{~h}$ by a factor of 45 and in the pineal where the activity was increased by a factor of 7 at $6 \mathrm{~h}$, whereas the increase in the PVN was 5-fold. At $6 \mathrm{~h}$, the medial basal hypothalamus was found to have an increased content of NOS measured in vitro and the collected cerebrospinal fluid (CSF) had increased concentrations of the NO metabolite, nitrate. These results indicate that the increase in iNOS mRNA was followed by de novo synthesis of iNOS that liberated NO into the tissue and also into the CSF. Presumably, LPS was bound to its receptors in the circumventricular organs and in the choroid plexus. These receptors, as in macrophages, activated DNA-directed IL-1ß mRNA synthesis which, in turn, caused the synthesis of IL- $1 \beta$. IL- $1 \beta$ then activated iNOS mRNA and synthesis (39).

How can neurons in the AN and PVN be activated since they are inside the blood brain barrier? In the case of the $\mathrm{AN}$, the neurons may have axons that project to the ME. These neurons may have LPS receptors on their cell surface which then induce IL- $B$ mRNA and IL- $1 \beta$ synthesis. IL- $1 \beta$ then induces iNOS mRNA followed by NO synthesis. Alternatively, LPS acting on its receptors may simultaneously induce IL- $\beta$ mRNA and iNOS mRNA.

Active transport mechanisms for IL-1 and other cytokines (40), and perhaps LPS, are present in the choroid plexus. The cells of the choroid plexus, on the basis of our results, must have LPS receptors on them. LPS then stimulates IL- $1 \beta$ and iNOS mRNA followed by synthesis of IL- $1 \beta$ and iNOS in the choroid plexus. LPS and IL- $1 \beta$ are then transported into the CSF. LPS is carried by CSF flow to the third ventricle, where it either crosses the ependyma or acts on terminals of PVN neurons in the ependyma to induce IL$1 \beta$ and iNOS mRNA.

This massive delayed increased NO production should further increase the effects of NO to maintain the pattern of hypothalamic hormone secretion already induced by LPS.
Unfortunately, the effects of inhibitors of NOS on these later stages in the response to LPS or infection have not yet been studied. Interestingly enough, in studies on LHRH release induced by NO, it has been shown that increasing concentrations of NO provided by release from sodium nitroprusside produce a bell-shaped dose-response curve in terms of LHRH release with values reaching a peak and then declining as the concentration of NO increases (41). Therefore, the massive increase in NO produced by iNOS several hours after injection of LPS might actually reduce the effects of NO on releasing hormone discharge below the peaks achieved earlier.

In addition to inducing production of proinflammatory cytokines such as IL-1, IL2, IL-6, and TNF $\alpha$, LPS also induces production of anti-inflammatory cytokines, such as IL-10 and IL-13 and IL-1 receptor antagonist in the brain, pituitary and pineal gland (42). In the periphery these inhibit the inflammatory response induced by the proinflammatory cytokines. Limited studies indicate that these anti-inflammatory cytokines antagonize the actions of the proinflammatory cytokines in the brain as well as the hypothalamic-pituitary response to infection (42).

The initial response to LPS is mediated by the constitutive nNOS present in the brain. There is no participation of the NO synthesized by iNOS in this initial response. Indeed, the initial response must be due to action on receptors for LPS on the endings of vagal afferents and also in areas where the blood brain barrier is not present, such as the choroid plexus, ME, organum vasculosum lamina terminalis, area postrema, and other circumventricular organs. Input to the hypothalamus from LPS by vagal afferents occurs at least in part by activation of the LC that sends NAergic axons to the hypothalamus to activate CRH release. LPS alters the release of the various hypophysiotropic hormones increasing release of $\mathrm{CRH}$ and vasopressin, 
GHRH and somatostatin, particularly in the rat, which overpowers the effect on the release of GHRH (41). LPS decreases release of the PRL-inhibiting hormone, dopamine, LHRH, TRH, but not FSHRF (37). All of these pathways involve participation of nNOS as described above (43).

CRH release from hypothalami incubated in vitro is controlled by muscarinic cholinergic receptors since it can be blocked by atropine $(44,45)$. The acetylcholine-producing interneurons in the hypothalamus release acetylcholine that stimulates a muscarinic-type receptor which in turn stimulates $\mathrm{CRH}$ release from the $\mathrm{CRH}$ neurons. nNOS has been located in neurons in the PVN of the hypothalamus. Stimulated $\mathrm{CRH}$ release can be blocked by $\mathrm{N}^{\mathrm{G}}$-monomethyl-L-arginine, a competitive inhibitor of all forms of NOS. Consequently, CRH release from the neurons in the PVN is stimulated by cholinergic neurons that synapse on these NOergic neurons to activate NOS. NOS synthesizes NO that diffuses into the CRH neurons and activates $\mathrm{CRH}$ release by activating cyclooxygenase I (COX I), leading to the generation of prostaglandin $\mathrm{E}_{2}$ from arachidonate (AA). Prostaglandin $\mathrm{E}_{2}$ activates $\mathrm{CRH}$ via activation of adenylyl cyclase and generation of cAMP. cAMP activates protein kinase $\mathrm{A}$ that induces exocytosis of $\mathrm{CRH}$ secretory granules into the hypophyseal portal vessels that then activates ACTH release from the corticotrophs of the anterior pituitary gland. NO activates not only COX but also lipoxygenase (LOX) that also plays a role in the activation of $\mathrm{CRH}$ release (46). NO also activates guanylyl cyclase that converts guanosine triphosphate into cGMP. cGMP is postulated to increase intracellular $\mathrm{Ca}^{2+}$ required to activate phospholipase $\mathrm{A}_{2}$ that converts membrane phospholipids into AA, the substrate for COX and LOX, permitting generation of prostaglandins and leucotrienes, respectively $(44,45)$.

Activation of CRH release can be blocked by the synthetic glucocorticoid dexametha- sone (46) and also by blockers of the three pathways of AA metabolism, such as clotrimazole that blocks epoxygenase, which in turn converts AA into epoxides; by indomethacin that inhibits $\mathrm{COX}$ and by 5'8'11eicisotrionoic acid that blocks LOX. Thus, CRH release is activated by the AA cascade (44). Alpha melanotropic-stimulating hormone also inhibits CRH release (46).

Cyclosporin inhibits CRH release as well (47), probably by inhibiting calcineurin. Calcineurin dephosphorylates NOS rendering it inactive.

Of the many proinflammatory cytokines, it has been shown that IL- $1 \alpha$ or $\beta$, TNF $\alpha$, IL6 and IL-2 can stimulate ACTH release from the anterior pituitary in vitro and in vivo (43). The principal action probably occurs, at least acutely, on the release of $\mathrm{CRH}$ and vasopressin from the hypothalamus but also there are clear effects at the pituitary level. There have been few studies on the mechanism of this direct pituitary action of cytokines, however, several cytokines such as IL-6 have been found to be produced in pituitaries and there is also nNOS present in the gland as indicated earlier. There are indications that NO participates in inhibiting the response of ACTH to vasopressin (23). Whether it plays a role in the stimulatory action of the various proinflammatory cytokines on ACTH secretion has not yet been studied.

In our studies LPS itself had no acute effect on ACTH release from hemianterior pituitaries in vitro (37). However, LPS induces cytokine production in the pituitary.

Cytokine production would be increased in a few hours and undoubtedly would modify the responses of the pituitary to the continued altered secretion of releasing and inhibiting hormones.

In addition to the proinflammatory cytokines which we have discussed extensively, it is now clear that there are a number of antiinflammatory cytokines, the first one to be discovered being the IL-1 receptor antago- 
nist, but IL-10 and IL-13 also play this role as indicated above. These are also induced in the brain by LPS and may play roles at hypothalamic and pituitary levels to diminish the response to the proinflammatory cytokines.

\section{References}

1. McCann SM (1988). Saga of the discovery of the hypothalamic releasing and inhibiting hormones. In: McCann SM (Editor), People and Ideas in Endocrinology. American Physiological Society, Bethesda, MD, 41-62.

2. McCann SM (1999). Hypothalamic releasing and inhibiting hormones. In: Adelman $\mathrm{G} \&$ Smith BH (Editors), Encyclopedia of Neuroscience. Elsevier, New York, 927930.

3. McCann SM \& Brobeck JR (1954). Evidence for a role of the supraopticohypophyseal system in regulation of adrenocorticotrophin secretion. Proceedings of the Society for Experimental Biology and Medicine, 87: 318-324.

4. McCann SM \& Fruit A (1957). Effect of synthetic vasopressin on release of adrenocorticotrophin in rats with hypothalamic lesions. Proceedings of the Society for Experimental Biology and Medicine, 96: 556-567.

5. Saffran $M \&$ Schally AV (1955). Release of corticotrophin by anterior pituitary tissue in vitro. Canadian J ournal of Biochemistry and Physiology, 33: 408-415.

6. Guillemin R \& Hearn WR (1955). ACTH release by in vitro pituitary: effect of Pitressin and purified arginine vasopressin. Proceedings of the Society for Experimental Biology and Medicine, 89: 365-367.

7. McCann SM (1957). The ACTH-releasing activity of extracts of the posterior lobe of the pituitary in vivo. Endocrinology, 60: 664-676.

8. McCann SM \& Porter J C (1969). Hypothalamic pituitary stimulating and inhibiting hormones. Physiological Reviews, 49: 240-283.

9. McCann SM \& Haberland P (1959). Relative abundance of vasopressin and corticotrophin-releasing factor in neurohypophyseal extracts. Proceedings of the Society for Experimental Biology and Medicine, 102: 319-325.

10. Dhariwal APS, Antunes-Rodrigues J, Reeser F, Chowers I \& McCann SM (1966). Purification of hypothalamic corticotrophin-releasing factor of ovine origin.
Proceedings of the Society for Experimental Biology and Medicine, 121: 8-12.

11. Rivier J, Spiess J \& Vale W (1983). Characterization of rat hypothalamic corticotropin-releasing factor. Proceedings of the National Academy of Sciences, USA, 80: 4851-4855.

12. McCann SM \& Ojeda SR (1996). The anterior pituitary and hypothalamus. In: Griffin JE \& Ojeda SR (Editors), Textbook of Endocrine Physiology. 3rd edn. Oxford University Press, New York, NY, 101-133.

13. McCann SM, Antunes-Rodrigues ] , Nallar $\mathrm{R} \&$ Valtin H (1966). Pituitary adrenal function in the absence of vasopressin. Endocrinology, 79: 1058-1064.

14. Yates FE, Russell $S M$, Dallman MF, Hedge GA, McCann SM \& Dhariwal APS (1971). Potentiation by vasopressin of corticotropin release induced by corticotropin-releasing factor. Endocrinology, 88: 315.

15. Gillies GE, Linton EA \& Lowry PJ (1982). Corticotropin releasing activity of the new $\mathrm{CRF}$ is potentiated several times by vasopressin. Nature, 299: 355-357.

16. Hedge GE, Yates MB, Marcus R \& Yates FE (1966). Site of action of vasopressin in causing corticotropin release. Endocrinology, 79: 328-340.

17. Ono N, Bedran de Castro J C, Khorram O $\&$ McCann SM (1985). Role of arginine vasopressin in control of ACTH and $\mathrm{LH}$ release during stress. Life Sciences, 36: 1779-1786.

18. Tilders FJ H, Schipper J , Lowry PJ \& Vermes I (1982). Effect of hypothalamus lesions on the presence of CRF-immunoreactive nerve terminals in the median eminence and on the pituitary-adrenal response to stress. Regulatory Peptides, 5: 77-84.

19. Gillies G \& Lowry PJ (1979). Corticotrophin releasing factor may be modulated by vasopressin. Nature, 278: 463-464.

20. Franci CR, Anselmo-Franci J A \& McCann SM (1997). Angiotensinergic neurons physiologically inhibit prolactin, growth hormone, and thyroid-stimulating hormone, but not adrenocorticotropic hormone, release in ovariectomized rats.
Peptides, 18: 971-976.

21. Gutkowska J, Antunes-Rodrigues J \& McCann SM (1997). Atrial natriuretic peptide in brain and pituitary gland. Physiological Reviews, 77: 465-515.

22. Franci CR, Anselmo-Franci J A \& McCann SM (1989). Opposite effects of central immunoneutralization of angiotensin II or atrial natriuretic peptide on luteinizing hormone release in ovariectomized rats. Neuroendocrinology, 51: 683-687.

23. Turnbull A \& Rivier C (1996). Corticotropin-releasing factor, vasopressin and prostaglandins mediate, and nitric oxide restrains, the hypothalamic-pituitary-adrenal response to acute local inflammation in the rat. Endocrinology, 137: 455-463.

24. McCann SM, Fruit A \& Fulford BD (1958). Studies on the loci of action of cortical hormones in inhibiting the release of adrenocorticotrophin. Endocrinology, 63: 29-42.

25. Chowers I \& McCann SM (1963). The effects on ACTH and gonadotrophin secretion of implants of gonadal steroids in the hypothalamo-hypophysial region. Israel Medical J ournal, 22: 420-432.

26. Reichlin S (1992). Neuroendocrinology. In: Foster DE \& Wilson JD (Editors), Textbook of Endocrinology. WB Saunders, Philadelphia, 135-220.

27. Goldstein LE, Rasmusson AM, Bunney BS \& Roth RH (1996). Role of the amygdala in the coordination of behavioral, neuroendocrine, and prefrontal cortical monoamine responses to psychological stress in the rat. J ournal of Neuroscience, 16: 4787-4798.

28. Arnsten AF, Mathew R, Ubriani R, Taylor J R \& Li BM (1999). Alpha-1 noradrenergic receptor stimulation impairs prefrontal cortical cognitive function. Biological Psychiatry, 45: 26-31.

29. Anselmo-Franci JA, Rocha-Barros VM, Franci CR \& McCann SM (1999). Locus ceruleus lesions block pulsatile LH release in ovariectomized rats. Brain Research, 833: 86-92.

30. Foote SL (1999). Locus coeruleus. In: Adelman G \& Smith BH (Editors), Encyclopedia of Neuroscience. Elsevier, New 
York, 1062-1063.

31. Bornstein SR \& Chrousos GP (1999). Clinical review: Adrenocorticotropin (ACTH)and non-ACTH-mediated regulation of the adrenal cortex: neural and immune inputs. J ournal of Clinical Endocrinology and Metabolism, 84: 1729-1736.

32. Ono N, Bedran de Castro J \& McCann SM (1985). Ultrashort-loop positive feedback of corticotropin-releasing factor (CRF) to enhance ACTH release in stress. Proceedings of the National Academy of Sciences, USA, 82: 3528-3531.

33. Ono N, Samson WK, McDonald J K, Lumpkin MD, Bedran de Castro JC \& McCann SM (1985). The effects of intravenous and intraventricular injection of antisera directed against corticotropin releasing factor (CRF) on the secretion of anterior pituitary hormones. Proceedings of the National Academy of Sciences, USA, 82: 7787-7790.

34. Ono N, Lumpkin MD, Samson WK, McDonald J K \& M CCann SM (1984). Intrahypothalamic action of corticotrophin-releasing factor (CRF) to inhibit growth hormone and $\mathrm{LH}$ release in the rat. Life Sciences, 35: 1117-1123.

35. Rivier C \& Vale W (1984). Influence of corticotropin-releasing factor on reproductive functions in the rat. Endocrinology, 114: 914-921.

36. Rivier C \& Vale W (1984). Corticotropinreleasing factor (CRF) acts centrally to inhibit growth hormone secretion in the rat.
Endocrinology, 114: 2409-2411.

37. Rettori V, Dees WL, Hiney J K, Lyson K \& McCann SM (1994). An interleukin-1-alpha-like neuronal system in the preoptichypothalamic region and its induction by bacterial lipopolysaccharide in concentrations which alter pituitary hormone release. Neuroimmunomodulation, 1: 251258.

38. McCann SM (2000). Nitric oxide. In: Fink $\mathrm{G}$ (Editor), Encyclopedia of Stress. Vol. 3. Academic Press, San Diego, CA, 53-61.

39. Wong $M-L$, Rettori $V$, Al-Shekhlee A, Bongiorno PB, Canteros G, McCann SM, Gold PW \& Licinio J (1996). Inducible nitric oxide synthase gene expression in the brain during systemic inflammation. Nature Medicine, 2: 581-584.

40. Banks WA, Kastin AJ, Huang W, Jaspan J B \& Maness LM (1996). Leptin enters the brain by a saturable system independent of insulin. Peptides, 17: 305-311.

41. Canteros G, Rettori V, Genaro A, Suburo A, Gimeno M \& McCann SM (1996). Nitric oxide synthase content of hypothalamic explants: Increased by norepinephrine and inactivated by NO and CGMP. Proceedings of the National Academy of Sciences, USA, 93: 4246-4250.

42. Wong $M-L$, Bongiorno $P B$, Rettori $V$, McCann SM \& Licinio J (1997). Interleukin (IL) 1ß, IL-1 receptor antagonist, IL-10, and IL-13 gene expression in the central nervous system and anterior pituitary during systemic inflammation: Pathophysi- ological implications. Proceedings of the National Academy of Sciences, USA, 94: 227-232.

43. McCann SM, Karanth S, Kamat A, Dees WL, Lyson K, Gimeno M \& Rettori V (1994). Induction by cytokines of the pattern of pituitary hormone secretion in infection. Neuroimmunomodulation, 1: 213.

44. Karanth S, Lyson K \& McCann SM (1993). Role of nitric oxide in interleukin 2-induced corticotropin-releasing factor release from incubated hypothalami. Proceedings of the National Academy of Sciences, USA, 90: 3383-3387.

45. Karanth S, Lyson K, Aguila MC \& McCann SM (1995). Effects of luteinizing-hormone-releasing hormone, $\alpha$-melanocytestimulating hormone, naloxone, dexamethasone and indomethacin on interleukin-2-induced corticotropin-releasing factor release. Neuroimmunomodulation, 2 : 166-173.

46. Lyson K \& McCann SM (1992). Involvement of arachidonic acid cascade pathways in interleukin-6-stimulated corticotropin-releasing factor release in vitro. Neuroendocrinology, 55: 708-713.

47. Karanth S, Lyson K \& McCann SM (1994). Cyclosporin A inhibits interleukin-2-induced release of corticotropin releasing hormone. Neuroimmunomodulation, 1: 82-85. 